\title{
AUOTOMATIC CLASSIFICATION OF POINT CLOUDS EXTRACTED FROM ULTRACAM STEREO IMAGES
}

\author{
M. Modiri ${ }^{\text {a* }}$, M. Masumi ${ }^{\text {b }}$, A. Eftekharic \\ a Professor at Malek Ashtar University of Technology, Esfahan, Iran- mmodiri@ut.ac.ir \\ ${ }^{\mathrm{b}}$ M.s degree of Information Technology Management,- mm.slm.masumi@ gmail.com \\ ${ }^{c}$ M.s of degree of Remote Sensing, Dept. of surveying and Geomatics engineering, University of Tehran, Tehran, Iran - \\ akrameftekary@gmail.com
}

Commission VI, WG VI/4

KEY WORDS: Point Clouds, Ultracam Images, point's classification, clustering, non-ground points, DEM

\begin{abstract}
:
Automatic extraction of building roofs, street and vegetation are a prerequisite for many GIS (Geographic Information System) applications, such as urban planning and 3D building reconstruction. Nowadays with advances in image processing and image matching technique by using feature base and template base image matching technique together dense point clouds are available. Point clouds classification is an important step in automatic features extraction. Therefore, in this study, the classification of point clouds based on features color and shape are implemented.

We use two images by proper overlap getting by Ultracam-x camera in this study. The images are from Yasouj in IRAN. It is semiurban area by building with different height. Our goal is classification buildings and vegetation in these points.

In this article, an algorithm is developed based on the color characteristics of the point's cloud, using an appropriate DEM (Digital Elevation Model) and points clustering method. So that, firstly, trees and high vegetation are classified by using the point's color characteristics and vegetation index. Then, bare earth DEM is used to separate ground and non-ground points.

Non-ground points are then divided into clusters based on height and local neighborhood. One or more clusters are initialized based on the maximum height of the points and then each cluster is extended by applying height and neighborhood constraints. Finally, planar roof segments are extracted from each cluster of points following a region-growing technique.
\end{abstract}

\section{INTRODUCTION}

Three-dimensional (3D) information of the earth one of the most important data on land management and its related events. Areal stereo imaging is one of the first methods that used in collection $3 \mathrm{D}$ information. Today, both improvements in camera technology and the rise of new matching approaches triggered the development of suitable software tools for image based 3D reconstruction by research groups and vendors of photogrammetric software. Based on dense pixel-wise matching, the photogrammetric generation of dense 3D point clouds and Digital Surface Models from highly overlapping aerial images has become feasible(Shorter and Kasparis 2009). These point clouds include of many artificial features as buildings, vegetation, pipe line, etc. that automatic features extraction from point clouds are interested by researchers.

In between, 3D building models are used for variety of applications such as urban planning, city modeling, disaster management, etc.

Accurate building models can be created manually by using aerial images, LIDAR data, building blueprints, and other data sources but often rely on costly and time consuming processing(Bandyopadhyay, van Aardt et al. 2013).

There are much research in classification and segmentation of point clouds that used to extract and detect vegetation and roof plan especially by LIDAR data but generating dense and high accurate point clouds by photogrammetric methods and aerial images is low price in comparison with LiDAR data(Omidalizarandi and Saadatseresht 2013). In most studies, methods of surface extraction can be categorized in two main groups. Firstly, surface parameters can be estimated directly by clustering or finding maximum parameter in the parameter space. Secondly, point clouds can be segmented on the basis of proximity of the point clouds or similarity measures like locally estimated surface normal(Vosselman, Gorte et al. 2004).

In this article, an algorithm is developed based on the color characteristics of the point's cloud, using an appropriate DEM (Digital Elevation Model) and points clustering method. Firstly, trees and high vegetation are classified by using the point's color characteristics and vegetation index. Then, bare earth DEM is used to separate ground and non-ground points.

The remaining not classified non-ground points are then divided into clusters based on height and local neighborhood. One or more clusters are initialized based on the maximum height of the points and then each cluster is extended by applying height and neighborhood constraints. Planar roof segments are extracted from each cluster of points following a region-growing technique. Planes are initialized using coplanar points as seed points and then grown using plane compatibility tests. If the estimated height of a point is similar to height different limit, or if it's normal distance to a plane is within a predefined limit, then the point is added to the plane. Once all the planar segments are extracted, the common points between the neighboring planes are assigned to the appropriate planes based on the plane intersection line, locality and the angle between the normal at a common point and the corresponding plane. Tree planes which are small in size and randomly oriented is removed by applying a rule-based procedure.

\footnotetext{
* Corresponding author
} 


\section{METHOLOGY}

The steps followed in the methodology used are shown in Figure 1.

The proposed method first separates vegetation from the input Point clouds data by applying vegetation index. Then ground and non-ground points are divided. The nonground points, including objects above the ground such as buildings and trees but trees are separated, are further processed for building extraction. We cluster points first and extract planar roof segments from each cluster of points using a region-growing technique in object space. The extracted segments are refined based on the relationship between each pair of neighboring planes. Points on the neighboring planar segments are accumulated to form individual building regions.

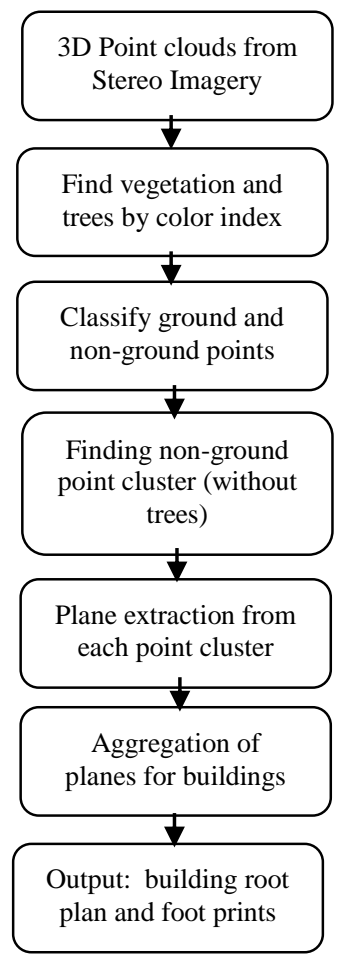

Figure1: the proposed building extraction method

\subsection{Vegetation and trees detection by using RGB texture in point clouds}

Since we used RGB images to extract point clouds, the three bands from the color image are utilized to calculate two different indices(Gitelson 2004).

Ratio Index (RI): This is the ratio of green radiance ( $\rho$ green) and the blue radiance ( $\rho$ blue).

$$
R I=\frac{\rho_{\text {green }}}{\rho_{\text {blue }}}
$$

Visible Atmospherically Resistant Index (VARI): This index is expressed as,

$$
V A R I=\frac{\rho_{\text {green }}-\rho_{\text {red }}}{\rho_{\text {green }}+\rho_{\text {red }}-\rho_{\text {blue }}}
$$

Both the index images have gray scale values between 0-255, which were then converted to the binary images based on a threshold value; this value was determined as a mid-range value between highest and lowest gray level for each individual index image. The intersection of these two index images produced a vegetation mask image(Bandyopadhyay, van Aardt et al. 2013).

\subsection{Finding non-ground points}

DEM (Digital Elevation Model) can be easily used to separate ground and non-ground points. If a bare-earth DEM is not available, one can be generated from the LIDAR point cloud data. We assume that the bare-earth DEM is given as an input to the proposed method. For each 3D point, the corresponding DEM height is used as the ground height $\mathrm{H}_{\mathrm{g}}$. A height threshold $\mathrm{T}_{\mathrm{h}}=$ $\mathrm{H}_{\mathrm{g}}+\mathrm{h}_{\mathrm{c}}$, where $\mathrm{h}_{\mathrm{c}}$ is a height constant that separates low objects from higher objects, is then applied to the data. In this study, $h_{c}$ $=1 \mathrm{~m}$ has been set(Awrangjeb and Fraser 2014).

\subsection{Points clustering}

All the non-ground points are now processed to generate clusters of points based on height.

Firstly, all these points are not belonged to any clusters. Considering the maximum data height as the current height, points at this height (within a specified tolerance) are found and one or more clusters are initialized depending on their locality. Then each of the cluster is extended until no points can be added to the cluster. Points in each cluster are marked so that they are not assigned to another cluster. Once all the clusters initialized from the current height are finalized, the points that are not yet assigned to any clusters are then processed to generate more clusters. The next current height will be the maximum height of these unassigned points.

Let the current maximum height be $h_{m}$ and the set of points that has similar height (i.e., within $h_{m} \pm T_{f}$, where $T_{f}=0.2$ $\mathrm{m}$ (Awrangjeb, Zhang et al. 2013) to allow the error in data generated heights) is $S_{c}$. One or more clusters $\xi_{i}$, where $i \geq 1$, are initialized using $S_{c}$ based on the locality. For a point $P \in \xi_{i}$, there is at least one neighboring point $\mathrm{Q}$ in the same cluster such that the 2D Euclidean distance $|\mathrm{P} . \mathrm{Q}| \leq \mathrm{H}_{\mathrm{d}}$. If $\mathrm{P}$ and $\mathrm{Q}$ are in two different clusters then $|\mathrm{P} . \mathrm{Q}|>\mathrm{H}_{\mathrm{d}}$.

In order to extend a cluster $\xi_{i}$, let $\mathrm{R}$ be a neighbor of $\mathrm{P}$, where $\mathrm{P} \epsilon \xi_{\mathrm{i}}$ but $\mathrm{R}$ has been neither assigned to any cluster. $\mathrm{R}$ is considered to be a neighbor of $P$, if $|P . R| \leq H_{d}$ and their height difference is within $\mathrm{V}_{\mathrm{d}}$. $\mathrm{R}$ is added to $\xi_{\mathrm{i}}$, which is iteratively extended until no $\mathrm{R}$ is found as a neighbor of $\mathrm{P}$ (Awrangjeb, Lu et al. 2014).

Eventually, an individual building, a roof plane or a roof section are represented by each of the extended clusters. Thereafter, an extended cluster $\xi_{\mathrm{i}}$ is considered a valid cluster if it is larger than $1 \mathrm{~m} 2$ in area. The area of $\xi_{\mathrm{i}}$ is thus roughly estimated by counting the number of black pixels multiplied by the pixel size.

\subsection{Roof Plan Extraction}

Segmentation is an efficient method for roof plan extraction. The segmentation method has two stages:

\subsubsection{Normal and Flatness estimation}

The normal for each point was estimated by fitting a plane to its neighboring points. The neighboring points can be selected based on $\mathrm{K}$ nearest neighbor (KNN) or fixed distance neighbor (FDN) methods(Rabbani 2006).

The estimation of normal vectors and flatness measure can be performed by eigen-analysis of the covariance matrix of the point positions. Let $p \in P$ be the sample point in the point clouds, where 
$p$ consists of $\mathrm{x}, \mathrm{y}$ and $\mathrm{z}$ coordinates, and $\bar{p}$ be the centroid of the neighborhood of $p$, i.e,

$$
\bar{p}=\frac{1}{\left|N_{p}\right|} \sum_{i \in N_{p}} p_{i}
$$

The $3 \times 3$ covariance matrix $C$ for the sample point $p$ is given by

$$
C=\frac{1}{\left|N_{p}\right|} \sum_{i \in N_{p}}\left(p_{i}-\bar{p}\right)\left(p_{i}-\bar{p}\right)^{T}
$$

If $\lambda_{0} \leq \lambda_{1} \leq \lambda_{2}$ are the eigenvalues sorted in the ascending order, then the eigenvector corresponding to the smallest eigenvalue, i.e., $\lambda_{0}$, defines the normal vector at any point $p$ (Bandyopadhyay, van Aardt et al. 2013). The flatness or surface variation 1 at any point $p$ can be estimated as:

$$
F=\frac{\lambda_{0}}{\lambda_{0}+\lambda_{1}+\lambda_{2}}
$$

\subsubsection{Region Growing}

This step uses the point normal and their flatness values to group points into smooth surfaces. Two constraints are followed in the region-growing algorithm(Rabbani 2006): I: Points belonging to a segment should be locally connected. The constraint is enforced by including the nearest neighbor in the region-growing process.

ii: Points belonging to a segment should form a smooth surface, i.e., surface normal of the points should vary within a predefined threshold $\left(\theta_{t h}\right)$. Region-growing starts with calculating the flatness $(\mathrm{F})$ of each point in the data set. Among all points, the one with minimum flatness is considered as a seed point and other points, based on their surface normal orientation, are iteratively added to the region(Bandyopadhyay, van Aardt et al. 2013).

Here we wanted to prevent over-segmentation of surfaces, but we also did not want all points to form a single segment. Thus, depending of the rooftop structure, we set a threshold angle between surface normal of points $\left(\theta_{t h}\right)$ at $10^{\circ}$.

Once all the planar segments are extracted, the common points between the neighboring planes are assigned to the appropriate planes based on the plane intersection line, locality and the angle between the normal at a common point and the corresponding plane. Tree planes which are small in size and randomly oriented is removed by applying a rule-based procedure(Awrangjeb, Lu et al. 2014).

\section{DATA SETS AND RESULTS}

\subsection{Data}

We use two images by proper overlap getting by Ultracam-x camera in this study. The images are from Yasouj in IRAN. It is semi-urban area by building with different height. Also Bare earth DEM by 1 meter resolution is used. Dense point clouds data are extracted from aerial stereo images. Orthophoto of studied area and point clouds of this are shown in Figure 2, 3.

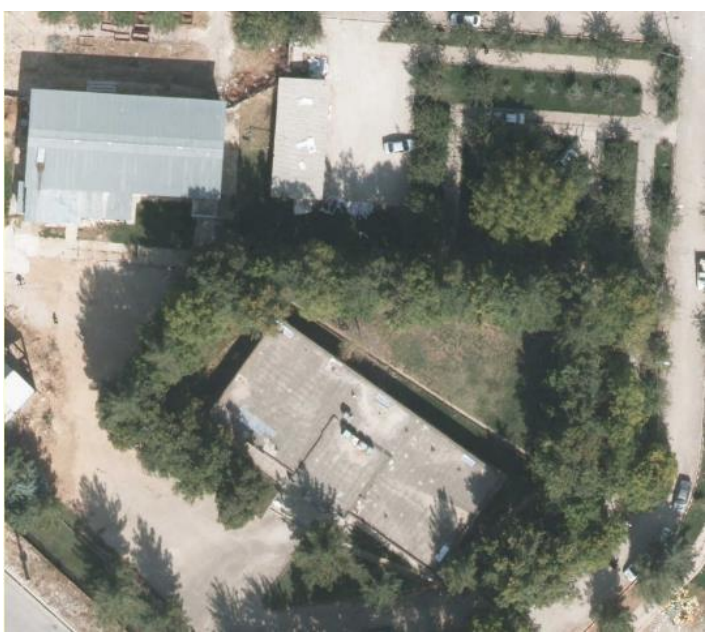

Figure 2: Orthophoto of studied area

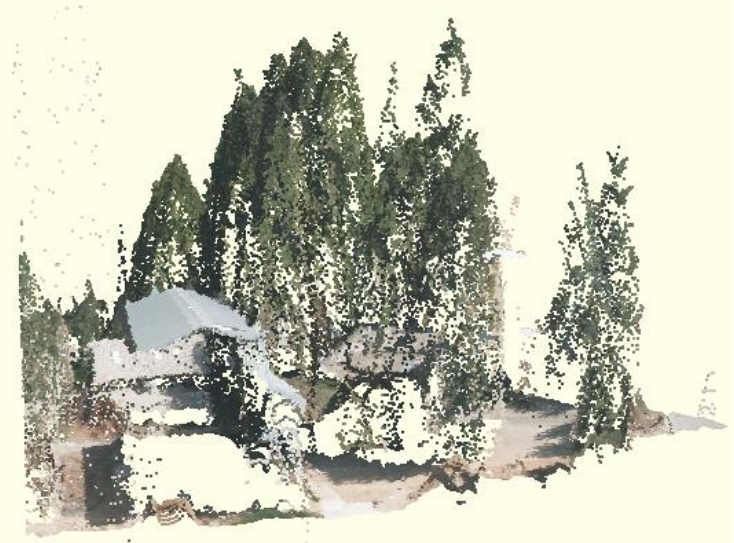

Figure 3: 3D view of point clouds

\subsection{Results}

By applying proposed method, at first step, vegetation and trees are extracted from point clouds by applying RI (Rational Index) indicator. We consider 1.2 for threshold value. The results of this step is shown in figure 4.

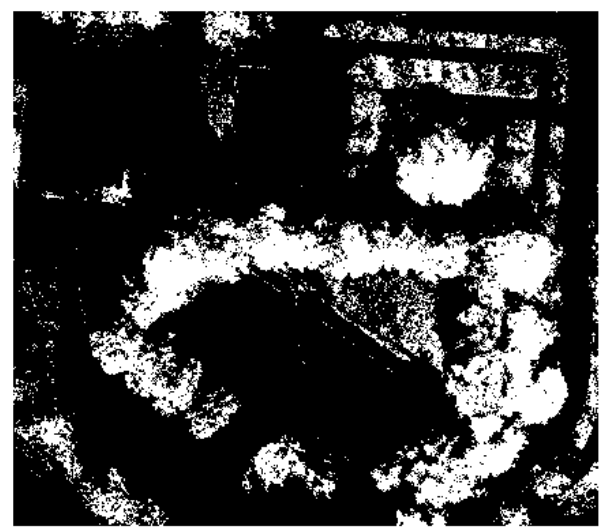

Figure4: white points are vegetation and trees in point clouds that extracted by RI and black points are unclassified points. 
At second step, bare earth DEM is used for separation ground and non-ground from data that process at previous step. The classified points in three class vegetation, ground and non-ground is presented as follow:

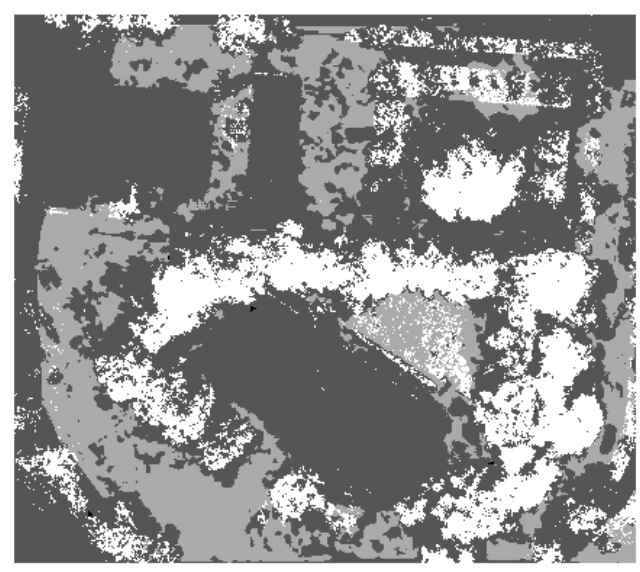

Figure5: points by grey color are ground points, black points are non-ground and white points are in ground vegetation and trees.

The next step in proposed methodology is clustering non-ground points by explained tactic in 2.3 section. Cluster points are shown in Figure 6.

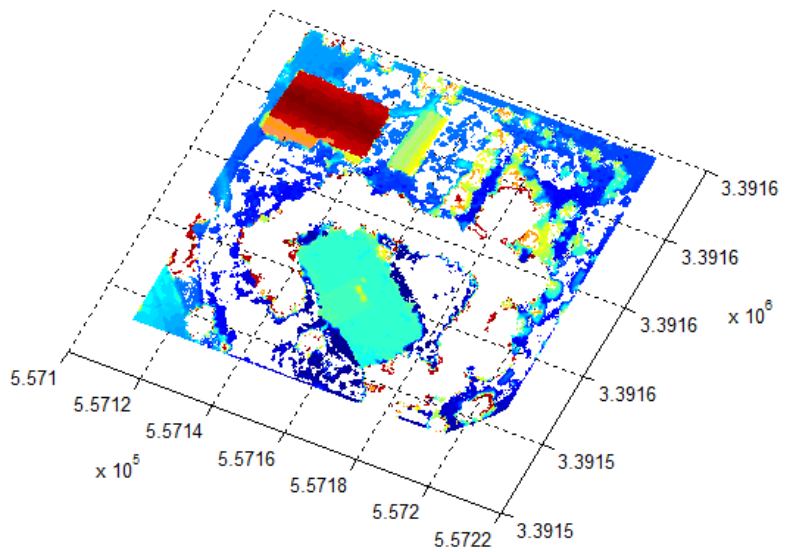

Figure6: points in different clusters are shown.

After applying segmentation method that described, roof plans are detected. The results shown in Figure 7.

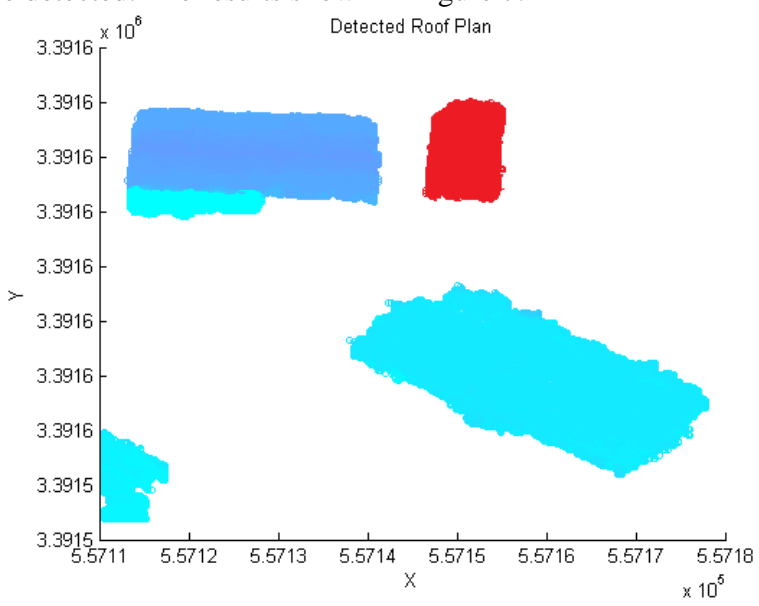

\section{CONCLUSIONS}

Our classification algorithm, based on the using dense point clouds extracted from areal stereo images by RGB texture, resulted in a good classification result for both buildings and vegetation in a semi-urban environment.

Visual comparison between ortho-image and results that shown in Figure 4 and 7, is demonstrated that the roof plans and vegetation have been identified with acceptable accuracy.

But we need ground truth for better evaluation. Also the results of this study can be compared by LIDAR data if it's available in the future studies. Finally, we want to implement buildings extraction model for extract boundary box of building for preparing data to input in GIS in the next studies.

\section{REFERENCES}

Awrangjeb, M. and C. S. Fraser (2014). "Automatic segmentation of raw LiDAR data for extraction of building roofs." Remote Sensing 6(5): 3716-3751.

Awrangjeb, M., et al. (2014). "Automatic building extraction from LIDAR data covering complex urban scenes." ISPRSInternational Archives of the Photogrammetry, Remote Sensing and Spatial Information Sciences 1: 25-32.

Awrangjeb, M., et al. (2013). "Automatic extraction of building roofs using LIDAR data and multispectral imagery." ISPRS Journal of Photogrammetry and Remote Sensing 83: 1-18.

Bandyopadhyay, M., et al. (2013). Classification and extraction of trees and buildings from urban scenes using discrete return LiDAR and aerial color imagery. SPIE Defense, Security, and Sensing, International Society for Optics and Photonics.

Gitelson, A. A. (2004). "Wide dynamic range vegetation index for remote quantification of biophysical characteristics of vegetation." Journal of plant physiology 161(2): 165-173.

Omidalizarandi, M. and M. Saadatseresht (2013). "SEGMENTATION AND CLASSIFICATION OF POINT CLOUDS FROM DENSE AERIAL IMAGE MATCHING." The International Journal of Multimedia \& Its Applications 5(4): 33.

Rabbani, T. (2006). Automatic reconstruction of industrial installations using point clouds and images, NCG Nederlandse Commissie voor Geodesie Netherlands Geodetic Commission.

Shorter, N. and T. Kasparis (2009). "Automatic vegetation identification and building detection from a single nadir aerial image." Remote Sensing 1(4): 731-757.

Vosselman, G., et al. (2004). "Recognising structure in laser scanner point clouds." International archives of photogrammetry, remote sensing and spatial information sciences 46(8): 33-38.

Figure7: Roof plan detected from point clouds. 